\title{
Fusarium Head Blight Development and Deoxynivalenol Accumulation in Wheat as Influenced by Post-Anthesis Moisture Patterns
}

\author{
Kelsey F. Andersen, Laurence V. Madden, and Pierce A. Paul
}

Department of Plant Pathology, The Ohio State University, Ohio Agricultural Research and Development Center, Wooster 44691. Accepted for publication 15 August 2014.

\begin{abstract}
Andersen, K. F., Madden, L. V., and Paul, P. A. 2015. Fusarium head blight development and deoxynivalenol accumulation in wheat as influenced by post-anthesis moisture patterns. Phytopathology 105:210-219.

Mist chamber experiments were conducted to quantify and model the effects of post-anthesis moisture on Fusarium head blight (FHB) index (IND) and deoxynivalenol (DON). Four mist treatments, one daily and three intermittent, were applied during an 8-day window immediately after anthesis, plus an untreated check. All intermittent mist treatments received moisture on 4 of the 8 days, but the distribution of the supplemental moisture during the treatment window varied among the treatments. Separate sets of spikes in each treatment were either spray or point inoculated with a spore suspension of Fusarium graminearum. Based on results from linear mixed-model analyses, mist treatment had a significant effect on arcsine-square root-transformed IND (arcIND) and $\log$-transformed DON $(\log \mathrm{DON})$ in spray-inoculated spikes but only a marginal effect on DON in point-inoculated spikes. The daily mist treatments (Mist1) consistently had the highest mean IND and DON but several of the 4-day intermittent mist treatments were not significantly different, particularly for point inoculations. Only Mist1 and one of the

(estimated diseased spikelets per spore) than the check; however, none of the treatments increased the rate of disease spread within the spike (based on visual symptoms) relative to the check. For all treatments, there was a significant, positive linear relationship between IND and $\log D O N$, with estimated mean regression slopes (rates of $\operatorname{logDON}$ increase per unit increase in IND) of 0.04 and $0.02 \log \mathrm{DON} \%^{-1}$ IND for the point- and spray-inoculated experiments, respectively. Mist treatment did not have a significant effect on the slope but had a significant effect on the intercept. The height of the regression line (logDON after adjusting for IND) was consistently higher for Mist2 than for Mist1 for both point- and sprayinoculated spikes. Estimated mean back-transformed DON at a fixed level of IND was 4.9 and $2.9 \mathrm{ppm}$ higher for Mist2 than Mist1 in the sprayand point-inoculation experiments, respectively. Generalized linear mixed models were used to estimate the risk of IND and DON exceeding critical thresholds, showing similar results among treatments in terms of estimated probabilities. The estimated probabilities of IND $\geq 10 \%$ at 20 days after inoculation and DON $\geq 2,5$, and $10 \mathrm{ppm}$ were not significantly different between Mist1 and Mist2. These results suggest that post-anthesis moisture patterns may play a role in DON exceeding critical thresholds even when FHB level are relatively low.
\end{abstract} intermittent mist treatments (Mist2; 2 days of mist at the beginning and end of the treatment window) had significantly higher infection efficiency
Additional keywords: logistic regression model.
Fusarium head blight (FHB) of wheat (Triticum aestivum), primarily caused by the fungal pathogen Fusarium graminearum and its associated toxins, is of great social and economic importance in most wheat-growing regions of North America. Losses due to FHB are primarily due to reductions in grain fill, yield, and seed quality, as well as mycotoxin contamination of grain. Major epidemics of FHB occurred in the 1990s, with economic losses estimated at nearly three billion dollars during that decade (36). Those epidemics and resulting losses were attributed to widespread adoption of reduced and minimum tillage, decreased crop diversity, increased acreage of host crops, and wet, humid weather conditions during anthesis and early grain fill $(3,9,19,20,36)$. Despite research efforts to develop FHB-resistant varieties and effective management strategies, outbreaks of FHB continued annually through the 2000s, with major epidemics in 2003 and 2010 (19).

Deoxynivalenol (DON), also known as vomitoxin, is the most abundant and problematic mycotoxin produced by $F$. graminearum. It is a member of the trichothecene class of fungalproduced mycotoxins and has been shown to inhibit protein syn

Corresponding author: P. A. Paul; E-mail: paul.661@ osu.edu

http://dx.doi.org/10.1094/PHYTO-04-14-0104-R

(C) 2015 The American Phytopathological Society thesis in eukaryotic cells, resulting in feed refusal, emesis, reproductive disorders, and growth retardation in mammals $(12,29)$. Because of these health concerns, the United States Food and Drug Administration has set a 2-ppm DON threshold for wheat grain (19). Consequently, grain with DON levels $>2$ ppm may be rejected completely or priced down at grain elevators, resulting in an economic loss for wheat producers.

Substantial gains in our understanding of the biology and epidemiology of FHB and DON have been made in recent years. It is now well known that weather variables, particularly those related to moisture, are important for several processes in the FHB infection cycle $(10,15,23,24,27)$. However, there is circumstantial and research-based evidence suggesting that the effects of moisture on FHB development and DON accumulation may depend on the characteristics and properties of this variable. The form (rainfall, surface wetness, or relative humidity $[\mathrm{RH}]$ ), amount, duration, pattern, and timing of moisture events during the infection cycle may all play a role in determining FHB intensity and the level of DON contamination. For instance, Nita et al. (22) demonstrated that supplemental moisture (mist irrigation) during anthesis led to higher levels of FHB and DON than similar amounts of supplemental moisture during grain fill. Culler et al. (7) observed that extended post-anthesis mist irrigation resulted in lower DON levels in grain than anticipated. Contrastingly, however, Cowger et al. (6) reported that increasing the duration of post-anthesis mist irrigation led to an increase in FHB and DON. The authors 
of the latter study attributed the apparent disparity to differences in the number of days with irrigation and the amount of water applied during each irrigation cycle.

A better understanding of the role of moisture (and other weather variables) on this disease-toxin complex is important for developing and refining prediction models to help guide FHB management and grain marketing decisions. DeWolf et al. (8) found that duration of precipitation and moderate temperatures during 7 days pre-anthesis were predictive of major FHB epidemics (defined as $>10 \%$ disease index). The accuracy of the predictive models they developed increased when post-anthesis weather variables were included as predictors. Similarly, investigating the role of weather patterns during pre- and post-anthesis windows on DON accumulation, Hooker et al. (14) also observed that rainfall during a 4- to 7-day window prior to anthesis was the most important predictor of DON. More recently, Shah et al. (33) attempted to further elucidate the role of pre- and post-anthesis weather variables on FHB development using a large dataset collected from 15 states over a period of 27 years. Out of 380 weather-based predictors, representing temperature, RH, and rainfall summarized for different pre- and post-anthesis time windows, 21 were selected and incorporated into 15 logistic regression models. Most of the weather-based predictors in the 15 models were derived from $\mathrm{RH}$ or temperature. Only one was based on rainfall. This led the authors to hypothesize that "relative humidity was better at characterizing moisture effect on FHB than other (moisture-related) variables".

It is very clear from empirical observations and designed experiments that extended periods of high $\mathrm{RH}$, particularly around anthesis, are critical for FHB development and DON accumulation. However, the role of variable moisture patterns of a fixed amount and duration during a specific window relative to anthesis is less certain. FHB may still develop and, most importantly, DON may still exceed the 2-ppm threshold in years with infrequent or discontinuous moisture patterns. Moreover, the disparity between FHB visual symptoms and corresponding DON accumulation $(5,34)$, sometimes observed in years with less-than-ideal conditions for major FHB epidemics, may also be associated with variable moisture patterns $(6,7)$. Controlled-environment experiments were designed to better understand the role of variable post-anthesis moisture patterns on FHB development, DON accumulation, and the association between FHB and DON. The specific objectives were to (i) evaluate the effect of intermittent post-anthesis moisture patterns on infection, disease development, and DON accumulation under controlled conditions and (ii) model the relationships between FHB and DON and estimate the probability of FHB and DON exceeding critical thresholds under variable moisture patterns.

\section{MATERIALS AND METHODS}

Preparation and maintenance of plants. Fungicide-treated seed of awnless, FHB-susceptible soft red winter wheat cultivar 'Hopewell' were sown in several batches between August and December 2012 in plastic trays with $10-\mathrm{cm}$ row spacing and allowed to germinate in the greenhouse for approximately 2 weeks. After germination, plants were fertilized with Osmocote (Scotts Miracle-Grow, Columbus, $\mathrm{OH}$ ) and allowed to vernalize in a cold $\left(3^{\circ} \mathrm{C}\right)$ room for 10 weeks. Plants were then transplanted to individual containers (Stuewe and Sons, Inc. Corvallis, OR) containing autoclaved silt loam and transferred to a greenhouse (average temperature: $26^{\circ} \mathrm{C}$ ). At approximately Feekes growth stage 7, plants were tied to bamboo stakes and excess tillers were trimmed to enhance proper spike development. At Feekes growth stage 8, plants were moved to a walk-in growth chamber (Conviron BDW40, Winnipeg, Manitoba, Canada) and placed under controlled conditions to help stimulate uniform development.
All growth chambers used in this study were programed to maintain a constant temperature of approximately $24^{\circ} \mathrm{C}\left( \pm 2^{\circ} \mathrm{C}\right)$ and $\mathrm{RH}$ close to $80 \%$ (ranging from 75 to $95 \%$ ), with a fixed photoperiod of $16 \mathrm{~h}$ of light and $8 \mathrm{~h}$ of darkness. Growth chamber conditions were monitored using built-in sensors, whereas conditions in the greenhouse and mist chambers were monitored using Watchdog (WatchDog 1450 Micro Station; Spectrum Technologies, Inc., Plainfield, IL) and Hobo (Hobo Micro Station; Onset Computer Corp. Bourne, MA) data loggers and sensors. Throughout the study, plants were watered once a day, and aphids and powdery mildew were controlled as needed using a standard insecticide (21.4\% imidacloprid [Marathon II]; OHP Inc., Mainland, PA) and a fungicide with little effect on FHB (50\% triadimefon [Bayleton 50]; Bayer CropScience, Research Triangle Park, NC).

Inoculum preparation and inoculation. Two separate sprayinoculated and two point-inoculated experiments were performed in mist and growth chambers. The spray-inoculation method was used to assess the effects of post-anthesis moisture on infection efficiency and subsequent disease development and DON accumulation, whereas the point-inoculation method was used to assess the effects of the moisture patterns on spike colonization (spread within the spike) as well as FHB development and DON accumulation, assuming an infection has already occurred. The two types of experiments were run either simultaneously or consecutively, depending on how the plants developed in the growth chamber, and were each repeated twice between December 2012 and May 2013.

All plants were inoculated at anthesis with a spore suspension consisting of a mixture of equal proportions of macroconidia from 10 Ohio isolates of $F$. graminearum (OHWAY1619, OHWAY627, OHWOO613, OHVAN4619, OHDEL3616, OHSHE6613, OHBUC6613, OHPAU2613, OHBUT611, and OHAUG621) of the 15-acetyldeoxynivalenol chemotype (D. Schmale, personal communication). Inoculum was produced by aseptically transferring mycelial plugs from Komada selective media to mung bean agar and incubating plates at room temperature under near ultraviolet lights, with a 12-h photoperiod. After 7 to 10 days, mycelia were removed and plates were transferred to a dark room for macroconidia development. After 2 weeks, macroconidia were harvested by adding $500 \mu \mathrm{l}$ of sterile deionized water to each plate and agitating using a rubber policeman to dislodge spores. Spore concentration was estimated using a hemacytometer, and diluted with deionized water to appropriate concentrations for immediate usage or storage at $0^{\circ} \mathrm{C}$ until used for inoculation.

For each replicate of each experiment, a set of 50 plants (10 per treatment) was inoculated at early anthesis (Feekes 10.5.1), when freshly extruded anthers were visible at the central spikelets of the spike. Working with 50 plants per block facilitated the process of obtaining plants (cohorts) at the same or close to the same growth stage, likely reducing some of the variability associated with differences in maturity commonly observed under natural field conditions. In the spray-inoculation experiments, a spore suspension containing 50,000 macroconidia of $F$. graminearum $/ \mathrm{ml}$ was applied to each spike using a spray bottle held approximately $30 \mathrm{~cm}$ from the spike. A single full spray was applied to one side of the spike; then, the spike was rotated $180^{\circ}$ and a second full spray was applied. Each spike received approximately $3.6 \times 10^{3}$ spores using this method. For point inoculation, the glume, lemma, and palea of a floret on the central spikelet were gently drawn back and, using a calibrated micropipette, $10 \mu \mathrm{l}$ of a spore suspension containing 10,000 spores $/ \mathrm{ml}$ was deposited directly into the floret (11). Each point-inoculated spike received approximately 100 spores.

Treatment application and data collection. Separate groups of inoculated plants were exposed to one of five mist treatments immediately following inoculation. Control (no-mist) plants were 
placed in a growth chamber with an average temperature of $24^{\circ} \mathrm{C}$ and average $\mathrm{RH}$ of approximately $80 \%$. The experimental design was a randomized complete block (with blocking in time), with experimental units replicated over three periods (blocks defined by batches of plants and cohorts of spikes at anthesis). There were five post-anthesis mist treatments imposed during an 8-day window immediately after early anthesis (inoculation): (i) mist every day (Mist1); (ii) 2-day mist, 4-day no mist, 2-day mist (Mist2); (iii) 2-day no mist, 4-day mist, 2-day no mist (Mist3); (iv) mist every other day (Mist4); and (v) no mist (Check). The treatment window length was determined based on the fact that previous studies have shown that weather variables summarized for this period were predicted of FHB and DON $(8,14,33)$. On a misting day, plants were placed in a mist chamber $(101$ by 218 by $101 \mathrm{~cm}$ ) and received $12 \mathrm{~h}$ of mist deployed for $90 \mathrm{~s}$ every $10 \mathrm{~min}$. These $12 \mathrm{~h}$ of high $\mathrm{RH}$ were followed by $12 \mathrm{~h}$ of no mist, to complete a full day. Average $\mathrm{RH}$ during the $12 \mathrm{~h}$ of mist was $89 \%$, with a maximum of $92 \%$ and minimum of $84 \%$. Average temperature was $21^{\circ} \mathrm{C}$. On days when a treatment required no mist, plants were placed in the same growth chamber as the control plants (at an average temperature of $24^{\circ} \mathrm{C}$ and average $\mathrm{RH}$ of approximately $80 \%$ ). At the end of the 8-day mist cycle, all plants were moved to a greenhouse (average temperature $27^{\circ} \mathrm{C}$ ) to await symptom development.

FHB index (mean percentage of diseased spikelets per spike) was rated on all 10 spikes in each experimental unit at 10 and 20 days (IND10 and IND20, respectively) after inoculation (i.e., 2 and 12 days, respectively, after the end of the mist cycle). After maturity and dry-down ( $<15 \%$ moisture), individual spikes from each experimental unit were hand harvested and threshed separately using a table-top thresher, and the grain was prepared and assayed for DON. Samples of grain ground using a laboratory mill (Laboratory Mill 3033; Perten Instruments, Springfield, IL) were sent for DON analysis to the U.S. Wheat and Barley Scab Initiative-funded mycotoxin testing laboratory at the University of Minnesota.

Data analysis. Post-anthesis moisture effect on FHB and DON. Because the effects of experiment and the experiment-treatment interaction were not statistically significant (based on the standard normal test) (Table 1), the two spray-inoculated experiments were pooled and the two point-inoculated experiments were pooled for analysis. Separate linear mixed models were fitted to the data from each type of experiment using the GLIMMIX procedure of
SAS (17) (SAS Institute, Cary, NC) to determine the effects of mist treatment on infection efficiency (IE), FHB index at 10 and 20 days after inoculation (IND10 and IND20, respectively), the rate of disease spread within the spike (rIND), and DON contamination of the grain. IE was defined as the mean number of diseased spikelets per 1,000 spores, and was estimated from IND10 in the spray-inoculated experiment as IE $=([\mathrm{IND} 10 \times 15] /$ 100)/3.6, where IND10 is as defined above (percent IND at 2 days after the end of the mist cycle), 15 is the mean number of spikelets per spike for the cultivar used in this study (Hopewell), and 3.6 is the mean number of spores applied per spike, divided by 1,000 . The rate of disease spread within the spike (IND/day) was estimated as the different between IND10 and IND20, divided by the number of days between the two assessments $($ IIND $=[$ IND20 - IND10]/10). IE was evaluated only in the spray-inoculated experiments, while rIND was evaluated only in the point-inoculated experiments.

Prior to analysis, disease index data were arcsine-square-roottransformed (arcIND) and DON data were log-transformed $(\log \mathrm{DON}+1)$ to stabilize variances. In all cases, the model fitted to data could be written as

$$
y_{i k}=\theta+\alpha_{i}+b_{k}+e_{i k}
$$

where $y_{i k}$ is the response (dependent variable; IE, arcIND10, arcIND20, rIND, or $\log$ DON) for the $i$ th mist treatment in the $k$ th block, $\theta$ is the constant (intercept), $\alpha_{i}$ is the fixed effect of the $i$ th mist treatment, $b_{k}$ is the random effect of the $k$ th block, and $e_{i k}$ is the residual. Separate analyses were performed for each response for the spray- and point-inoculated experiments, and treatment means were compared using contrast and lsmestimate statements in PROC GLIMMIX.

IND/DON relationships. Relationships between DON and IND20, as influenced by mist treatments, were modeled for both point- and spray-inoculated experiments. IND20 was used as a continuous covariate and treatment as a class variable, and linear mixed-model regression analyses were performed using PROC GLIMMIX of SAS (17). The effect of treatment and the continuous variable are correlated and there is no unique ordering of variables within the mixed model. Thus, type 3 hypothesis tests were used. All random effect terms from equation 1 were maintained but the equation was modified by adding terms for IND20

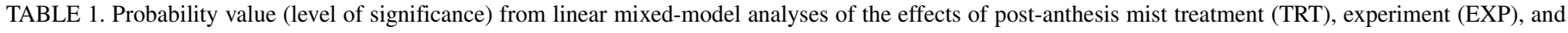

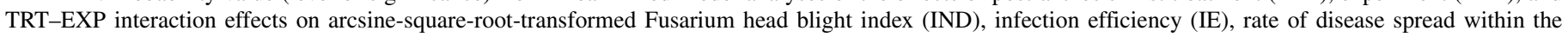

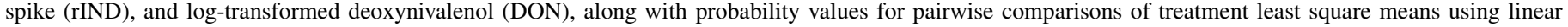
contrast for spray- and point-inoculated mist chamber experiments ${ }^{\mathrm{a}}$

\begin{tabular}{|c|c|c|c|c|c|c|c|c|c|c|}
\hline \multirow[b]{2}{*}{ Comparison $^{\mathrm{b}}$} & \multicolumn{5}{|c|}{ Spray } & \multicolumn{5}{|c|}{ Point } \\
\hline & IND10 & IND20 & IE & DON & DONa & IND10 & IND20 & rIND & DON & DONa \\
\hline Mist1 vs. Check & $<0.001$ & 0.003 & $<0.001$ & $<0.001$ & 0.116 & 0.052 & 0.519 & 0.046 & 0.625 & 0.865 \\
\hline Mist 2 vs. Check & 0.005 & 0.013 & 0.019 & $<0.001$ & 0.003 & 0.084 & 0.424 & 0.776 & 0.036 & 0.051 \\
\hline Mist 3 vs. Check & 0.028 & 0.038 & 0.091 & 0.022 & 0.351 & 0.446 & 0.570 & 0.337 & 0.958 & 0.726 \\
\hline Mist4 vs. Check & 0.040 & 0.043 & 0.147 & 0.018 & 0.271 & 0.133 & 0.707 & 0.623 & 0.577 & 0.662 \\
\hline Mist 2 vs. Mist 1 & 0.025 & 0.488 & 0.014 & 0.326 & 0.079 & 0.808 & 0.156 & 0.081 & 0.012 & 0.041 \\
\hline Mist 3 vs. Mist1 & 0.004 & 0.240 & 0.002 & 0.092 & 0.346 & 0.212 & 0.938 & 0.267 & 0.588 & 0.601 \\
\hline Mist4 vs. Mist1 & 0.003 & 0.218 & 0.001 & 0.110 & 0.444 & 0.622 & 0.311 & 0.119 & 0.300 & 0.551 \\
\hline TRT & $<0.001$ & 0.031 & $<0.001$ & $<0.001$ & 0.022 & 0.258 & 0.546 & 0.287 & 0.099 & 0.012 \\
\hline EXP & 0.744 & 0.642 & 0.746 & 0.784 & $\ldots$ & 0.952 & 0.286 & 0.874 & 0.724 & $\ldots$ \\
\hline $\mathrm{EXP} \times \mathrm{TRT}$ & 0.433 & 0.796 & 0.464 & 0.085 & $\ldots$ & 0.596 & 0.402 & 0.585 & 0.673 & $\ldots$ \\
\hline
\end{tabular}

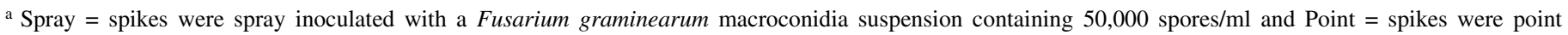
inoculated by pipetting $10 \mu \mathrm{l}$ of a macroconidia suspension containing 10,000 spores/ml into the central spikelet of each spike. IND10 = mean proportion of diseased spikelets per spike rated at 10 days after anthesis, IND20 = rated at 20 days after anthesis, IE $=$ spikelets diseased per 1,000 spores, rIND $=$ percent IND/day between 10 and 20 days after anthesis [(IND20 - IND10)/10], DON = DON content of harvested grain $($ ppm), and DONa $=$ adjusted log-transformed DON.

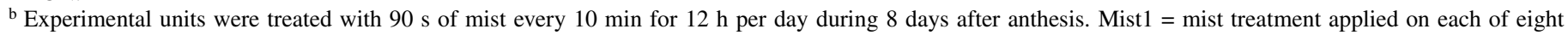
consecutive days after anthesis; Mist 2 = mist applied only on the first and last 2 days of the 8-day window; Mist $3=$ mist applied only on the middle 4 days and no mist on the first and last 2 days of the treatment window; and Mist 4 = mist applied every other day, beginning on the first day of the treatment window. Check $=$ no mist. 
and the IND20-treatment interaction. The model fitted to the data was

$$
y_{i j k}=\theta+\alpha_{i}+\delta X_{j}+\Delta_{i} X_{j}+b_{k}+e_{i j k}
$$

where $y_{i j k}$ is the response $(\operatorname{logDON}), X_{j}$ is the $j$ th observation of the covariable IND20, $\delta$ is the (main) effect of the covariable, $\Delta_{i}$ is the interaction effect of the covariable (effect of the mist treatment on the relationship between $y$ and $X$ ), and $\theta, \alpha_{i}, b_{k}$, and $e_{i j k}$ are the same as defined above.

Risk of IND and DON exceeding critical thresholds. Separate analyses were performed to estimate the expected probability of FHB IND exceeding 10\% (P_IND10; used here and in FHB risk assessment as the definition of an FHB epidemic) (33) for pointand spray-inoculated experiments, and P_DON2, P_DON5, and P_DON10 (probabilities of DON exceeding 2, 5, and $10 \mathrm{ppm}$, respectively) for the spray-inoculated experiment. The disease and DON status of each of 10 spikes rated per experimental unit was used to estimate the response variable $(Y)$. For P_IND10, $Y$ was defined as $\mathrm{D} / \mathrm{N}$, where $\mathrm{D}=$ number of spikes per experimental unit with IND $\geq 10 \%$ and $\mathrm{N}=$ total number of spikes rated (10). For P_DON2, P_DON5, and P_DON10, the DON concentration of the grain harvested from each of the 10 individual spikes from each experimental unit was quantified and used to obtain separate sets of $\mathrm{D}$ values (and correspondingly, $Y$ values) for DON $\geq 2,5$, and $10 \mathrm{ppm}$ (number of spikes in each experimental unit with DON concentration $\geq 2,5$, and $10 \mathrm{ppm}$, respectively).

$Y$ was assumed to have a conditional binomial distribution; therefore, generalized linear mixed models (GLMMs) were fitted to the data as described by Gbur et al. (13). Models were fitted to the logit link function ( $\eta$, also known as the linear predictor) of $Y$ as

$$
\eta_{i k}=\log \left[\pi_{i k} /\left(1-\pi_{i k}\right)\right]=\theta+\alpha_{i}+b_{k}+d_{i k}
$$

where $\log \left[\pi_{i k} /\left(1-\pi_{i k}\right)\right]$ is the logit link function and $\pi_{i k}$ is the probability of IND $\geq 10 \%$ (or $\mathrm{DON} \geq 2,5$, or $10 \mathrm{ppm}$ ) given the $i$ th treatment. All other terms in the model are the same as defined above. After fitting the models, treatments were compared on the logit scale (differences between log odds), and then P_IND10, P_DON2, P_DON5, and P_DON10 were estimated for each mist treatment from the corresponding $\eta$ value, using the inverse link function

$$
\pi_{i k}=e^{\eta_{i k} /\left(1+e^{\left.\eta_{i k}\right)}\right.}
$$

\section{RESULTS}

Post-anthesis moisture effect on FHB and DON. At 10 days post-inoculation (DPI), for the experiment utilizing the spray inoculation method, Mist1 displayed the highest mean IND (34.3\%), while Mist2 had the highest mean IND (19.3\%) among the three treatments that employed an intermittent mist regimen (Fig. 1A). Mist3, Mist4, and the nonmisted check had mean IND of, 14.9, 13.4, and 5.0\%, respectively (Fig. 1A). At 20 DPI, a similar numerical trend was observed, with Mist1, Mist2, Mist3, Mist4, and the check having mean IND of 50.5, 43.3, 40.1, 39.3, and 22.1, respectively (Fig. 1C). Interestingly, for this experiment, even without the highest level of visual symptoms (IND), Mist2 had the highest mean DON (75.01 ppm), followed by Mist1, Mist3, Mist4, and the check, with means of 59.53, 23.97, 23.23, and 8.75 ppm, respectively (Fig. 1E).

For the point-inoculation experiment, Mist1 and Mist2 had the highest mean IND at 10 DPI (19.3 and $19.4 \%$, respectively), while Mist3, Mist4, and the check had means of 14.0, 17.9, and $12.8 \%$, respectively (Fig. 1B). A slightly different trend was ob- served among treatments at 20 DPI. Mist2 and Mist 4 had the highest mean IND20 (40.3 and 37.6\%, respectively), with Mist1, Mist3, and the unmisted check having means of 30.0, 31.0, and $35.2 \%$, respectively (Fig. 1D). Interestingly, as with the sprayinoculation experiment, the highest mean DON was seen in plants exposed to Mist2 (15.70 ppm) (Fig. 1F). Mist1, Mist3, Mist4, and the check had mean DON levels of 4.96, 8.12, 8.62, and 6.05, respectively (Fig. 1F).

Linear mixed-model analysis was performed to assess the effects of post-anthesis mist treatment on transformed IND10, IND20, and DON (arcIND10, arcIND20, and $\log D O N$ ), and rIND. Pairwise treatment comparisons were carried out using the lsmestimate statements and are summarized in Table 1. All treatments were contrasted with both the check and the daily mist reference treatment (Mist1) for both the point- and spray-inoculation methods. For the spray-inoculation experiment, the effect of mist treatment was significant for all measured response, with all mist treatments having significantly higher arcIND10, arcIND20, and $\log$ DON than the check. However, only Mist1 and Mist2 had significantly higher IE than the check. When compared with Mist1, all three of the discontinuous mist treatments had significantly lower IE and arcIND10; however, arcIND20 and $\log$ DON were not significantly different between daily and discontinuous post-anthesis mist treatments. Interestingly, for the point-inoculation experiment, there were no significant treatment effects on arcIND10, arcIND20, rIND, or logDON (Table 1).

FHB/DON relationships. The above analysis shows the direct and indirect influence of treatment on $\log \mathrm{DON}$, and one cannot tell whether a change in DON is due to the treatment effect on disease intensity with corresponding change in DON or to direct effect of the treatment on toxin levels (that does not involve change in disease intensity). Therefore, linear mixed-model regression analyses were used to model relationships between IND and $\log$ DON as influenced by post-anthesis mist treatments (Table 2; Fig. 2). In all cases, $\log D O N$ was significantly affected by mist treatments and IND (as a covariate) but not by interactions between the covariate and the qualitative treatment factors. The $P$ values (levels of significance) for the mist treatment-IND interactions were 0.603 and 0.258 for the spray- and point-inoculated experiments, respectively. Without statistically significant interactions, a generic model for the relationship between expected $[\mathrm{E}(\log \mathrm{DON})]$ and IND can be written as

$$
E(\log \mathrm{DON})_{i}=\alpha_{i}+\delta \cdot \mathrm{IND}_{i}
$$

where $E(\log \mathrm{DON})$ is the expected value, $\alpha_{i}=$ intercept for each treatment, and $\delta=$ common slope (rate of $\log \mathrm{DON}$ increase per unit increase in IND) for each experiment (Table 2). The rate of $\log \mathrm{DON}$ increase per unit increase in IND was $0.02 \operatorname{logDON} \%^{-1}$ for the point-inoculated experiment and $0.04 \operatorname{logDON} \%{ }^{-1}$ for the spray-inoculated experiment.

Estimated intercepts (height of regression lines) were compared among treatments, with emphasis on comparisons between daily mist treatments and discontinuous treatments (Table 1; Fig. 2). The intercept was significantly higher for Mist 2 (mist only on the first and last 2 days of the 8-day post-anthesis treatment window) than Mist1 (mist on all 8 days) at $P \leq 0.05$ for the pointinoculated experiment (Table 1; Fig. 2B) and at $P \leq 0.08$ for the spray-inoculated experiment (Table 1; Fig. 2A). The level of $\log \mathrm{DON}$ at a fixed level of IND for Mist1 was not significantly different from Mist3, Mist4, or the check in either experiment (Table 1). Estimated back-transformed DON at IND zero was $8.4 \mathrm{ppm}$ for Mist2 compared with 3.5 for Mist1 for spray inoculation. The corresponding values for point inoculation were 4.8 and $1.9 \mathrm{ppm}$, respectively, for the same two treatments.

Risk of FHB and DON exceeding critical thresholds. GLMMs were fitted to the data from both experiments and probabilities 
were estimated as described above as measures of the risk of IND and DON exceeding critical levels. In all cases, the lsmestimate statements in PROC GLIMMIX were used to compare treatments on the link scale (logit, which is an estimate of the log odds) and then the results were reported on the data scale (probability) following conversion using the inverse link function (Fig. 3). P_IND10 was evaluated at both 10 and 20 DPI (Fig. 3). As would be expected, for both types of experiments, P_IND10 was higher at 20 DPI (Fig. 3C and D) than at 10 DPI (Fig. 3A and B). For the spray-inoculation experiment, there was a significant treatment effect on P_IND10 (on the logit scale) at both 10 and 20 DPI $(P<$ $0.001)$, with all mist treatments having higher $\mathrm{P} \_$IND10 values than the check (Fig. 3A and C). Among the mist treatments, Mist1 and Mist 3 were significantly different from Mist4 at 10 DPI (Fig. $3 \mathrm{~A})$ but the mist treatments were not significantly different from each other at 20 DPI (Fig. 3C). All mist treatments had between a 45 and $86 \%$ chance of resulting in IND $>10 \%$ at 10 DPI (Fig. $3 \mathrm{~A}$ ), and $>78 \%$ chance at 20 DPI (Fig. 3C). Trends in the point- inoculated experiment were very similar to those observed in the spray-inoculated experiment; however, the effect of treatment was only statistically significant at 10 DPI $(P=0.019)$ but not at 20 DPI $(P=0.110)$. At $10 \mathrm{DPI}$, the check had the lowest P_IND10 (0.61), being significantly lower than Mist1 and Mist3 (Fig. 3B). All mist treatments had P_IND10 >0.70 at 10 DPI (Fig. 3B) and $>0.80$ at $20 \mathrm{DPI}$ (Fig. 3D).

Probability of DON exceeding thresholds of 2 (P_DON2), 5 (P_DON5), and $10 \mathrm{ppm}$ (P_DON10) for the spray-inoculation experiment was also evaluated. For all thresholds, the effect of mist treatment was statistically significant (Fig. 4). In all cases, the check had the lowest estimated probabilities $(<0.20)$. Mist 1 , Mist2, Mist3, and Mist4 all had mean P_DON2 values $>0.60$, with no statistical difference among Mist1, Mist2, and Mist3 (Fig. 4A). A similar trend was apparent for P_DON5 and P_DON10 (Fig. 4B and C). Interestingly, for all threshold levels, Mist1 had the highest estimated probability, with Mist2 not being significantly different. This suggests that this particular post-anthesis

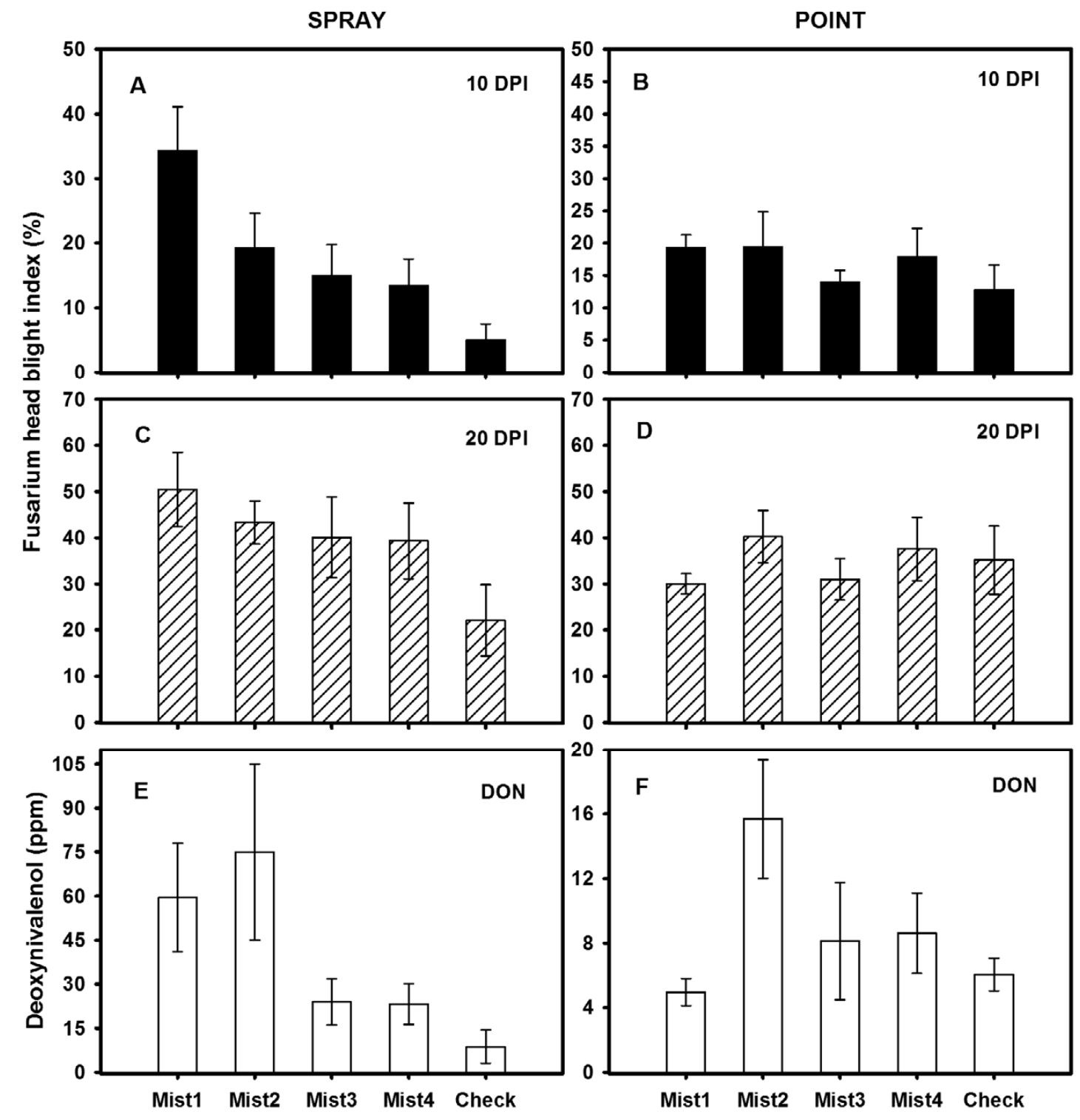

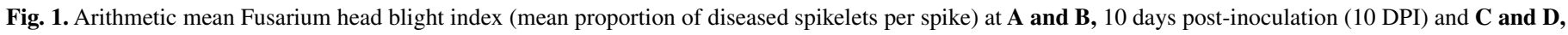

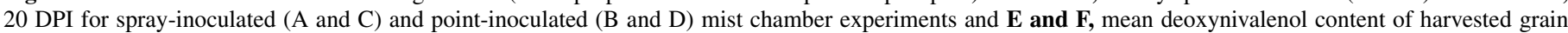

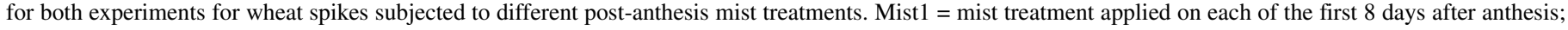

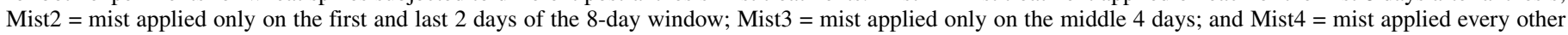

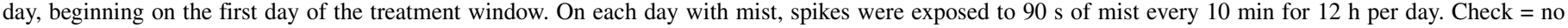

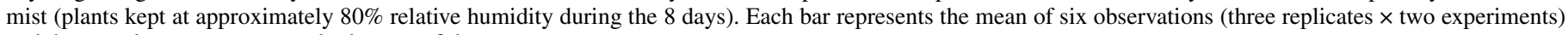
and the error bars represent standard errors of the mean. 
mist pattern that received 4 days less mist than Mist1 may still yield equally high probabilities of exceeding critical DON thresholds (Fig. 4).

\section{DISCUSSION}

The role of moisture on FHB development and DON accumulation has been widely investigated, with several studies providing invaluable information pertaining to the effects of moisture amount, duration, and timing relative to anthesis on this disease and its associated toxin $(6,7,15,22)$. Most of these previous studies were conducted under field conditions with supplemental irrigation, and few focused specifically on the distribution or pattern of moisture within a fixed window pre- or post-anthesis. As alluded to in several of the aforementioned publications, field experiments are subject to the confounding effects of natural weather conditions, variation in anthesis among tillers within experimental units, and variation in natural inoculum density, among other factors. Although there are numerous positive aspects to investigating the effects of moisture on FHB and DON under field conditions, uncontrolled (or even uncontrollable) factors may affect the consistence of results among studies, often leading to contrasting findings and conclusions. Here, we investigated the effects of moisture within a fixed post-anthesis window under controlled conditions, which allowed us to minimize the effects of several confounding factors, inoculate and subject plants to mist treatments at a fixed and known growth stage, and control the amount, duration, and pattern of mist treatments. To our knowledge, these experiments are the first to look into the effects of specific postanthesis moisture patterns on IND and DON under controlled conditions.

Our results showed that post-anthesis mist (amount and pattern) during the first 8 days after anthesis (Feekes 10.5.1) had an effect on IND in the spray-inoculated study but had little discernable effect in the point-inoculated study. In the former study, the difference in mean IND between the daily 8-day mist treatment and the 4-day treatments, regardless of the mist pattern, were only significant at 10 DPI (2 days after the end of the treatment window). By 20 DPI, the treatment effect in terms of the amount of moisture had disappeared, with the 8- and 4-day treatments having similar levels of disease but still significantly higher levels than the check. This suggests that the post-anthesis moisture treatments evaluated herein likely had an effect on penetration

TABLE 2. Regression coefficients from linear mixed-models analyses of relationship between Fusarium head blight index (IND) as a continuous covariate and log-transformed deoxynivalenol (DON) content of harvest wheat grain as influenced post-anthesis mist treatment ${ }^{\mathrm{a}}$

\begin{tabular}{lccccc}
\hline & \multicolumn{2}{c}{ Spray } & & \multicolumn{2}{c}{ Point } \\
\cline { 2 - 3 } \cline { 5 - 6 } Factor $^{\mathrm{b}}$ & Intercept & SE & & Intercept & SE \\
\hline Mist1 & 1.50 & 0.655 & & 1.05 & 0.337 \\
Mist2 & 2.24 & 0.596 & & 1.76 & 0.408 \\
Mist3 & 1.11 & 0.570 & & 1.22 & 0.344 \\
Mist4 & 1.18 & 0.565 & & 1.25 & 0.389 \\
Check & 0.69 & 0.449 & & 1.11 & 0.372 \\
Slope $^{\text {c }}$ & 0.04 & 0.011 & & 0.02 & 0.009 \\
\hline
\end{tabular}

a Spikes were spray inoculated with a Fusarium graminearum macroconidia suspension containing 50,000 spores/ml or point inoculated by pipetting $10 \mu \mathrm{l}$ of a macroconidia suspension containing 10,000 spores $/ \mathrm{ml}$ into the central spikelet of each spike. $\mathrm{SE}=$ standard error.

${ }^{\mathrm{b}}$ Mist $1=$ mist on each of eight consecutive days after anthesis; Mist $2=$ mist only on the first and last 2 days of the treatment window; Mist $3=$ mist only on the middle 4 days and no mist on the first and last 2 days of the treatment window; and Mist4 = mist every other day, beginning on the first day of the treatment window. Check $=$ experimental units that received no mist treatment.

${ }^{c}$ Intercepts $(\log D O N$ when IND $=0)$ and slopes (the rate of increase in $\log \mathrm{DON}$ per unit increase in IND) were estimated from linear mixed-model regression analysis. and infection, which take place during the first few hours after anthesis (28). Once infection had occurred, mimicked by the point-inoculation process (where the necessary spore germination on and penetration of the infection court were bypassed), 4 days of post-anthesis mist, with any pattern, was sufficient under the conditions of this study (with temperature within a favorable range for FHB) to yield comparable levels of disease to 8 consecutive days with mist. Bai (2) also showed that, when spikes were point-inoculated, the number of consecutive days with moisture (16 h/day) immediately after inoculation had less of an effect on FHB severity (comparable with IND, as defined in our study), and argued that, although high moisture was required for infection, it was less important for fungal spread within the spike (as measured by IND).

As reviewed by Magarey et al. (18), the effect of interrupted moisture on pathogen infection and disease development has been the subject of investigation in many pathosystems. They categorized plant pathogens into three broad groups-sensitive, moderate, and insensitive to interrupted moisture-based on whether and by how much interrupted moisture reduced the level of disease they caused. Insensitive pathogens were described as those for which it took $>24 \mathrm{~h}$ of dry conditions (RH $<95 \%)$ for infection (based on visual symptom) to be reduced by $50 \%$. There is little information in the literature on the effects of interrupted moisture on FHB development but our results suggest that, at

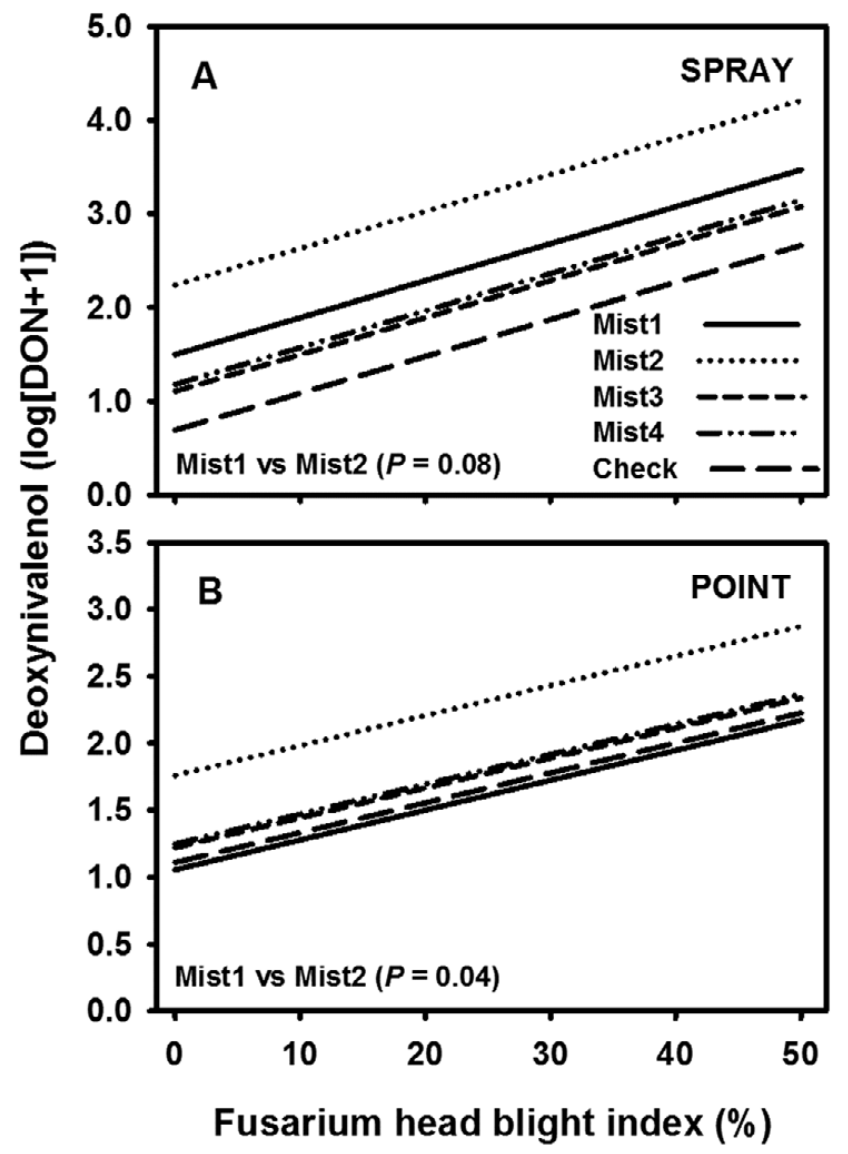

Fig. 2. Relationship between Fusarium head blight index (\%) and log-transformed deoxynivalenol (DON) $(\mathrm{DON}+1)$ as influenced by post-anthesis mist treatments for mist chamber $\mathbf{A}$, spray-inoculated and $\mathbf{B}$, point-inoculated experiments. Mist $1=$ mist treatment applied on each of the first 8 days after anthesis; Mist $2=$ mist applied only on the first and last 2 days of the 8-day window; Mist $3=$ mist applied only on the middle 4 days; and Mist $4=$ mist applied every other day, beginning on the first day of the treatment window. On each day with mist, spikes were exposed to $90 \mathrm{~s}$ of mist every $10 \mathrm{~min}$ for $12 \mathrm{~h}$ per day. Check $=$ no mist (plants kept at approximately $80 \%$ relative humidity during the 8 days). The insert shows level of significance for the difference in mean $\log \mathrm{DON}$ between Mist 1 and Mist 2 at a fixed level of index. 
favorable temperatures, $F$. graminearum may be "insensitive" to interrupted supplemental moisture in terms of its ability to infect and cause FHB. Based on data from our spray-inoculation study, only Mist 1 (daily mist for $12 \mathrm{~h}$ /day during the 8-day window) and Mist2 (mist only on the first and last 2 days of the treatment window) had significantly higher IE (diseased spikelets per 1,000 spores) than the check. Both of these treatments had at least 2 days with supplemental moisture ( $12 \mathrm{~h} /$ day) immediately after inoculation. Mist4, the treatment with 1 day with supplemental moisture (12 h/day) immediately after inoculation followed by a dry day ( $24 \mathrm{~h}$ at $\approx 80 \% \mathrm{RH}$ ) did not have significantly higher IE than the check.

These results suggest that, for $F$. graminearum, two or more consecutive days with high moisture (mist for $12 \mathrm{~h} /$ day) immediately after inoculation increased IE relative to the no-mist treatment; however, the pattern of mist interruption did not affect final disease index (at $20 \mathrm{DPI}$ ) when compared with the treatment with mist on each of the 8 days after anthesis. This is somewhat contrary to the conclusion drawn by Andersen (1) from a similar study, who observed that IE (defined as percent spikelet infected at 10 DPI following spray inoculation at anthesis) and FHB severity (comparable with IND, as defined in our study) were highest (93 and 52\%, respectively) when spikes were exposed to $48 \mathrm{~h}$ ( 2 days) of continuous wetness immediately after inoculation. However, both responses were reduced by $>50 \%$ when spikes were subjected to a short 6-h wet period immediately after inoculation, followed by a 4- or 8-day dry period, before being subjected to a second but longer wet period $(48 \mathrm{~h})$. Disagreement between our results and those reported by Andersen (1) may be due to the fact that the number of consecutive hours of high $\mathrm{RH}$ before the dry period was much longer in our study. As discussed by Magarey et al. (18), sensitivity to interrupted moisture may depend on when the dry period $(<95 \% \mathrm{RH})$ occurs relative to the wet period and the length of the wet period before the dry period, as well as the length of the dry period.

The observed response to interrupted post-anthesis moisture may, in part, explain some situations in which higher levels of FHB than expected based on predictions made by the current FHB risk assessment tool (www.wheatscab.psu.edu) are observed in the field. In some locations or years with infrequent rainfall, relatively high levels of FHB are observed even though the risk tool predicts low to moderate risk of the disease (personal observation). It is clear that multiple factors, including weather conditions outside of the 7-day pre-anthesis time window considered by the risk tool, late infection, and failure to correctly identify flowering date (an entry required to correctly use the risk tool), may all lead to misclassification of disease risk. However, our results suggest that post-anthesis moisture patterns may also be playing a role. We evaluated the estimated risk of IND exceeding $10 \%$ (a threshold used to denote an epidemic in the current disease forecasting system) (33) as influenced by postanthesis moisture patterns. For the spray-inoculation experiment, both at 10 and 20 DPI, all treatments had significantly higher probability of exceeding the $10 \%$ IND (P_IND10) threshold than the check. At $10 \mathrm{DPI}$, Mist1 had the highest probability, with Mist2 and Mist3 not being significantly different from Mist1. However, by 20 DPI, P_IND10 was $>0.8$ in all mist treatments but remained $<0.6$ in the check, indicating that the risk of an FHB epidemic may be just as high for 8 days with high moisture (12 h/day with $\mathrm{RH} \approx 90 \%)$ as with 4 days with high moisture, regardless of how it is distributed during the 8-day post-anthesis window evaluated here. In fact, in the point-inoculation experiment, P_IND10 was $>0.8$ in all treatments, including the check, suggesting that, once infection had occurred, $\mathrm{RH}$ close to $80 \%$
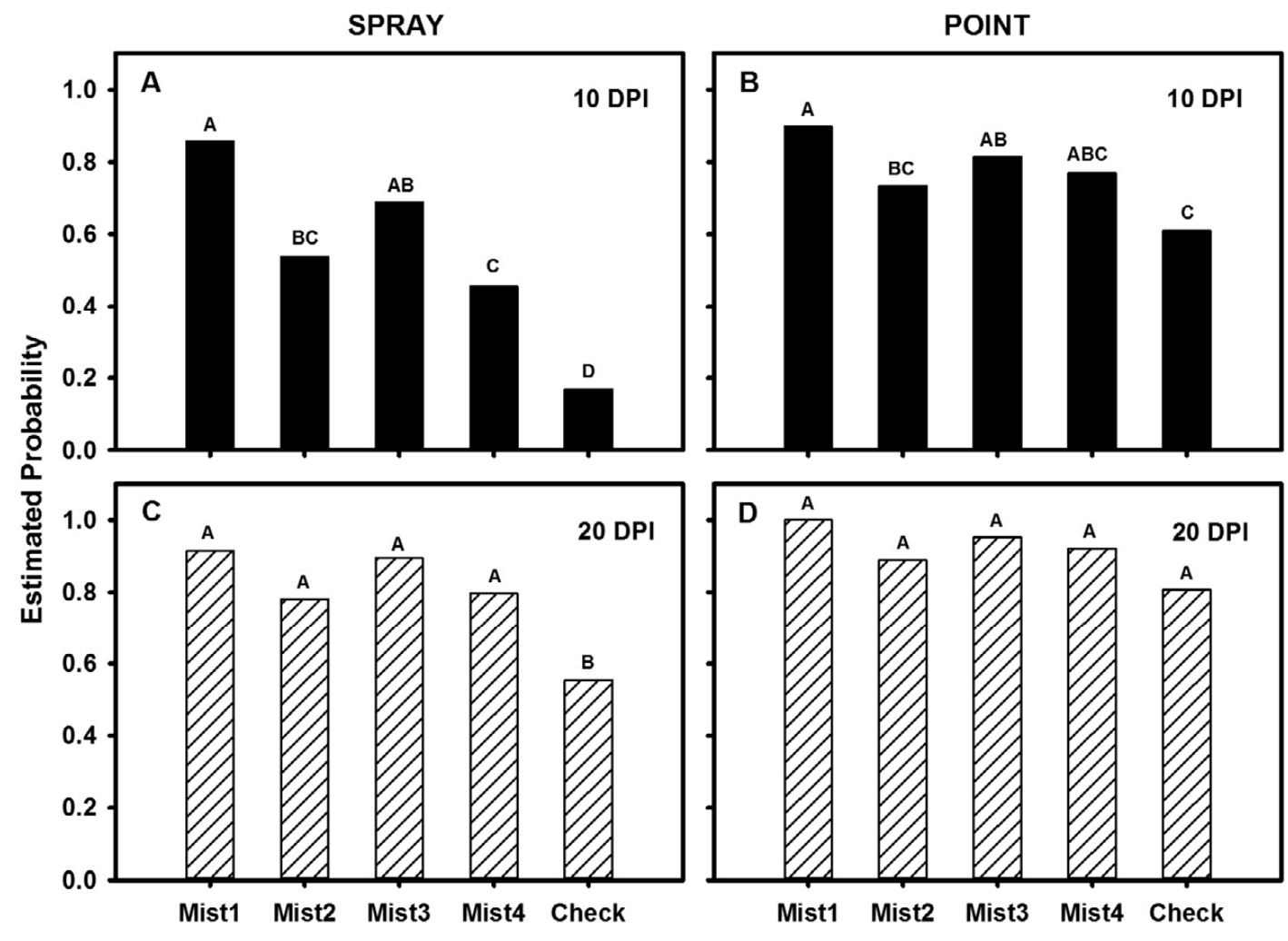

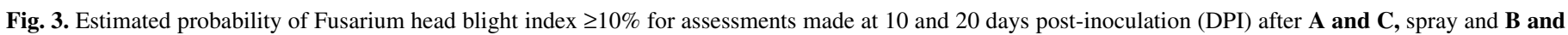

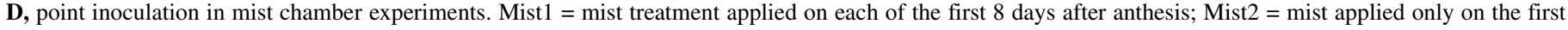

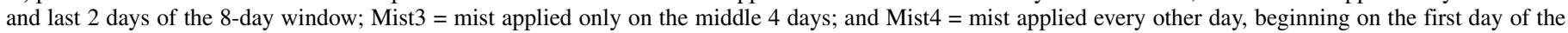

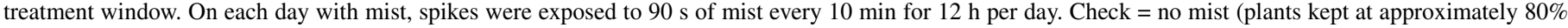

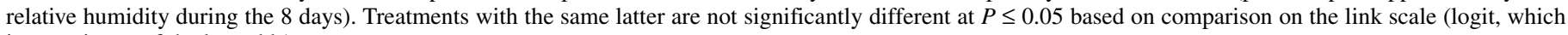
is an estimate of the $\log$ odds). 
(condition of the no-mist check) was sufficient to increase the risk of FHB. These results are consistent with empirical observations made by Shah et al. (33) in an investigation of the role of pre- and post-anthesis weather on FHB. Of the final 15 FHB risk prediction models they selected (based on prediction accuracy), 9 included some form of $\mathrm{RH}>80 \%$ as an important predictor of FHB epidemics (IND > 10\%). Of these nine models, eight were driven by RH-based predictors summarizing conditions during the first 5 to 12 day after anthesis. The average misclassification rate (a measure of prediction accuracy) of these models was lower than that of the currently used FHB risk tool, which is largely based on $\mathrm{RH}>90 \%$ (33).

The effect of interrupted post-anthesis moisture on DON contamination of $F$. graminearum-infected wheat grain and the risk of DON exceeding critical thresholds were also investigated herein, building on previously generated information $(6,7,16,22)$. Due to the positive association between IND and $\operatorname{DON}(4,21,25,26)$ and, consequently, the confounding effect of IND on DON, it is often not easy to discern whether DON responses to post-anthesis moisture as reported in several previous studies $(6,7,16,22)$ were actually due to the treatment effect on disease index with corresponding change in DON or due to direct effect of the treatment on the toxin itself (that does not involve change in disease intensity). Therefore, in the current investigation, linear mixed-model regression analysis was conducted to allow comparison of DON after adjusting for IND and to compare the IND/DON relationship among mist treatments. As previously reported $(4,21,25,26)$, there was a significant positive linear relationship between IND and DON (on the log-transformed scale), with DON levels increasing as IND increased. In none of our experiments were the rates (slopes of the regression line) at which $\log \mathrm{DON}$ increased per unit IND increase influenced by mist treatments; however, the intercepts (heights of the regression line) were.

The height of the regression line $(\operatorname{logDON}$ at a fixed level of IND) was generally higher for one particular type of intermittent moisture pattern - the one with two consecutive days of mist, followed by four consecutive days without supplemental moisture, and then another 2 days of mist (Mist2) - than for the daily mist treatment (Mist1). This particular moisture pattern seemed to affect some biological process which results in increased DON even at a fixed level of visual symptoms. Because the analytical approach used in this study allowed us to control for the confounding effects of visual symptoms on DON response to moisture treatment, it would be reasonable to assume that the observed differences in DON were not necessarily solely due to difference in disease index. In fact, Mist2 was not always the treatment with the highest mean IND yet this treatment had the highest mean $\log$ DON at a fixed level of IND in both studies. Post-anthesis moisture-influencing factors likely contributed to DON increase in a manner that was somewhat independent of changes in visual symptoms.

Mist 2 also had an equally high probability of DON exceeding 2-, 5-, and 10-ppm (P_DON2, P_DON5, and P_DON10) thresholds as Mist1 (everyday mist). In fact, all four mist treatments had higher risk of DON exceeding the three thresholds than the check but Mist 2 and Mist 1 had similarly high DON risk probabilities at all three threshold levels. These findings suggest that, even if moisture is discontinuous, as long as there are at least 4 days of high mean RH (close to $90 \%$ ), then the risk of DON exceeding critical economically important limits may be high. Furthermore, if there is an interrupted moisture pattern similar to our Mist2 treatment, with 2 days of high RH conditions immediately after anthesis, followed by 4 days of less-humid conditions (close to $80 \%$ ), the risk of DON exceeding 2, 5, and $10 \mathrm{ppm}$ may be just as high as when there is daily high $\mathrm{RH}$ during that critical initial week after anthesis.

The fact that Mist1 and Mist2 had the highest IE, similar mean arcIND at 20 DPI, similar estimated probabilities of IND and
DON exceeding the tested thresholds, and Mist2 had higher adjusted DON (on the log scale) than Mist1 suggests that interrupted moisture may contribute to disproportionately high levels of DON for a given level of visual symptoms. This is a fairly common occurrence in wheat fields and several factors have been suggested as being responsible, including weather conditions, post-anthesis infection (5), cultivar-specific reaction (34), and combinations of these factors (35). Although the mechanisms involved in DON response to Mist2 are unclear, we can still speculate and formulate hypotheses for future studies. One such hypothesis is that the result may have been due to a stress response by the pathogen to the imposed wet-dry post-anthesis

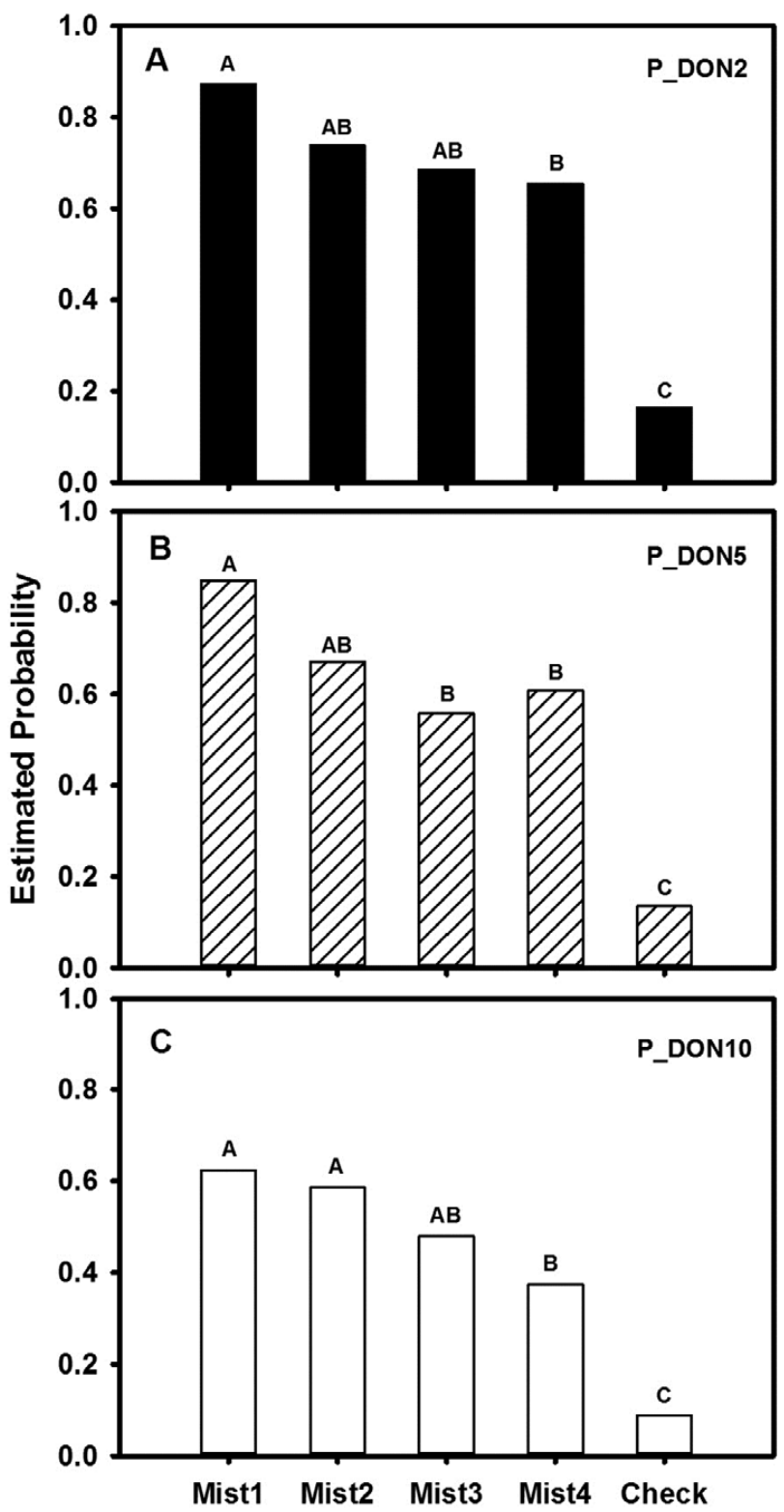

Fig. 4. Estimated probability of deoxynivalenol (DON) contamination of harvested grain $\mathbf{A}, \geq 2, \mathbf{B}, 5$, and $\mathbf{C}, 10 \mathrm{ppm}$ (P_DON2, P_DON5, and P_DON10, respectively) for spray-inoculated spikes exposed to different postanthesis mist treatments. Mist1 = mist treatment applied on each of the first 8 days after anthesis; Mist $2=$ mist applied only on the first and last 2 days of the 8-day window; Mist3 = mist applied only on the middle 4 days of the window; and Mist 4 = mist applied every other day, beginning on the first day of the treatment window. On each day with mist, spikes were exposed to $90 \mathrm{~s}$ of mist every $10 \mathrm{~min}$ for $12 \mathrm{~h}$ per day. Check = no mist (plants kept at approximately $80 \%$ relative humidity during the 8 days). Treatments with the same latter are not significantly different at $P \leq 0.05$ based on comparison on the link scale (logit, which is an estimate of the log odds). 
(post-infection) moisture cycle. Increased mycotoxin production in response to stress is fairly common among mycotoxigenic fungal genera such as Penicillium, Fusarium, and Aspergillus (31). For Mist2, the immediate introduction of a fairly extended dry period (4 days of $80 \% \mathrm{RH}$ ) following an initial wet period ( 2 days each with $12 \mathrm{~h}$ of mist and RH close to $90 \%$ ) may have triggered a stress response that caused the pathogen to increase DON production to enhance colonization. With mist on each of the 8 days, such a response likely did not occur in spikes subjected to Mist1. The effect of our post-inoculation wet-dry cycle may have some similarity to that seen in $F$. graminearum stress response to warm-cold temperature cycles described by Ryu and Bullerman (30). Results from their study showed that in vitro DON production by $F$. graminearum was highest when an extended period of cold stress followed a period of moderate temperatures. Willyerd (35) observed higher levels of DON when a resistant wheat cultivar was inoculated and incubated at 15 than at $22^{\circ} \mathrm{C}$, and also speculated that this response was due to a cold temperature-induced stress response.

Key findings from this study were that (i) post-anthesis moisture patterns within the first 8 days after anthesis were likely important for infection (based on spray inoculations) but, once infection had occurred, IND was similar regardless of amount and pattern of mist (within the ranges tested here); (ii) intermittent post-anthesis moisture patterns, particularly if wet days are separated by dryer days in the middle of the period, could lead to higher levels of DON at a fixed level of IND, compared with daily high moisture during the 8-day post-anthesis window; and (iii) the risk of IND and DON exceeding critical thresholds may be just as high for 8 consecutive days with high moisture as for 4 days of interrupted high moisture, particularly if wet days are interrupted by dryer days. These findings helped to improve our understanding of moisture effect on FHB and DON (at least for a susceptible soft red winter wheat cultivars with characteristics similar to those of Hopewell) and would be of value for ongoing efforts to develop and refine risk prediction models for this disease-toxin complex. However, it is important to point out that further research is needed to determine whether moderately resistant cultivars, particularly those with high levels of type I (resistance to infection) and type II (resistance to spread) resistance (32) will respond similarly to the types of moisture treatments investigated here. Future studies involving the analysis of fungal biomass (through quantitative polymerase chain reaction, for instance) and the Tri5 gene expression profile in susceptible and resistant cultivars under different moisture treatments and moisture stress may help to further explain why some intermittent moisture patterns led to elevated DON (without necessarily increasing the level of visual symptoms) relative to daily high moisture within the treatment window evaluated in this study.

\section{ACKNOWLEDGMENTS}

This investigation is based upon work partially supported by the United States Department of Agriculture through the U.S. Wheat \& Barley Scab Initiative (agreement number 59-0206-9-071) and the Ohio Small Grains Marketing Program. Salaries and research support for K. F. Andersen, P. A. Paul, and L. V. Madden were provided by state and federal funds to the Ohio Agricultural Research and Development Center. We thank B. James, J. D. Salgado, K. Davies, D. D’Angelo, J. Engle, and $\mathrm{K}$. Willyerd for assisting with establishment and maintenance of plants in the greenhouse. Any opinions, findings, conclusions, or recommendations expressed in this publication are those of the authors and do not necessarily reflect the view of the United States Department of Agriculture.

\section{LITERATURE CITED}

1. Andersen, A. L. 1948. The development of Gibberella zeae head blight of wheat. Phytopathology 38:595-611.

2. Bai, G. 1995. Scab of wheat: epidemiology, inheritance of resistance, and molecular markers linked to cultivar resistance. Ph.D. thesis, Department of Botany and Plant Pathology, Purdue University, West Lafayette, IN.

3. Bai, G., and Shaner, G. 1994. Scab of wheat: Prospects for control. Plant Dis. 78:760-766.

4. Bai, G., Plattner, R., Desjardins, A., and Bolb, F. 2001. Resistance to Fusarium head blight and deoxynivalenol accumulation in wheat. Plant Breed. 120:1-6.

5. Cowger, C., and Arrellano, C. 2010. Plump kernels with high deoxynivalenol linked to late Gibberella zeae infection and marginal disease conditions in winter wheat. Phytopathology 100:719-728.

6. Cowger, C., Patton-Özkurt, J., Brown-Guedira, G., and Perugini, L. 2009. Post-anthesis moisture increased Fusarium head blight and deoxynivalenol levels in North Carolina winter wheat. Phytopathology 99:320-327.

7. Culler, M. D., Miller-Garvin, J. E., and Dill-Macky, R. 2007. Effect of extended irrigation and host resistance on deoxynivalenol accumulation in Fusarium-infected wheat. Plant Dis. 91:1464-1472.

8. De Wolf, E. D., Madden, L. V., and Lipps, P. E. 2003. Risk assessment models for wheat Fusarium head blight epidemics based on within-season weather data. Phytopathology 93:428-435.

9. Dill-Macky, R., and Jones, R. K. 2000. The effect of previous crop residues and tillage on Fusarium head blight of Wheat. Plant Dis. 84:7176.

10. Dufault, N. S., De Wolf, E. D., Lipps, P. E., and Madden, L. V. 2006. Role of temperature and moisture in the production and maturation of Gibberella zeae perithecia. Plant Dis. 90:637-644.

11. Engle, J. S., Madden, L. V., and Lipps, P. E. 2003. Evaluation of inoculation methods to determine resistance reactions of wheat to Fusarium graminearum. Plant Dis. 87:1530-1535.

12. Foroud, N. A., and Eudes, F. 2009. Trichothecenes in cereal grains. Int. J. Mol. Sci. 10:147-173.

13. Gbur, E. E., McCarter, K. S., Durham, S., Young, L. J., Christman, M., West, M., and Kramer, M. 2012. Analysis of generalized linear mixed models in the agricultural and natural resource sciences. American Society of Agronomy, Soil Science Society of America, Crop Science Society of America, Madison, WI.

14. Hooker, D. C., Schaafsma, A. W., and Tamburic-Ilincic, L. 2002. Using weather variables pre- and post-heading to predict deoxynivalenol content in winter wheat. Plant Dis. 86:611-619.

15. Lacey, J., Bateman, G. L., and Mirocha, C. J. 1999. Effects of infection time and moisture on development of ear blight and deoxynivalenol production by Fusarium spp. in wheat. Ann. Appl. Biol. 134:277-283.

16. Lemmens, M., Buerstmayr, H., Krska, R., Schuhmacher, R., Grausgruber, H., and Ruckenbauer, P. 2004. The effect of inoculation treatment and long-term application of moisture on Fusarium head blight symptoms and deoxynivalenol contamination in wheat grains. Eur. J. Plant Pathol. 110:299-308.

17. Littell, R. C. Milliken, G. A., Stroup, W. W., Wolfinger, R. D., and Schabenberger, O. 2006. SAS for Mixed Models, 2nd ed. SAS Institute, Cary, NC.

18. Magarey, R. D., Sutton, T. B., and Thayer, C. L. 2005. A simple generic infection model for foliar fungal plant pathogens. Phytopathology 95:92100 .

19. McMullen, M., Bergstrom, G., De Wolf, E., Dill-Macky, R., Hershman, D., Shaner, G., and Van Sanford, D. 2012. A unified effort to fight an enemy of wheat and barley: Fusarium head blight. Plant Dis. 96:17121728 .

20. McMullen, M., Jones, R., and Gallenberg, D. 1997. Scab of wheat and barley: A re-emerging disease of devastating impact. Plant Dis. 81:13401348.

21. Mesterházy, A. 2002. Role of deoxynivalenol in aggressiveness of Fusarium graminearum and F. culmorum in resistance to Fusarium head blight. Eur. J. Plant Pathol. 108:675-684.

22. Nita, M., Tilley, K., De Wolf, E., and Kuldau, G. 2005. Effects of Moisture during and after anthesis on the development of Fusarium head blight of wheat and mycotoxins production. Pages 125-128 in: Proc. 2005 Natl. Fusarium Head Blight Forum. S. M. Canty, J. Lewis, L. Siler, and R. W. Ward, eds. Hilton Milwaukee City Center, Milwaukee, WI.

23. Paul, P. A., El-Allaf, S. M., Lipps, P. E., and Madden, L. V. 2004. Rain splash dispersal of Gibberella zeae within wheat canopies in Ohio. Phytopathology 94:1342-1349.

24. Paul, P. A., Lipps, P. E., De Wolf, E., Shaner, G., Buechley, G., Adhikari, T., Ali, S., Stein, J., Osborne, L., and Madden, L. V. 2007. A distributed lag analysis of the relationship between Gibberella zeae inoculum density on wheat spikes and weather variables. Phytopathology 97:1608-1624.

25. Paul P. A., Lipps, P. E., and Madden, L. V. 2005. Relationship between visual estimates of Fusarium head blight intensity and deoxynivalenol accumulation in harvested wheat grain: A meta-analysis. Phytopathology 95:1225-1236

26. Paul, P. A., Lipps, P. E., and Madden, L. V. 2006 Meta-analysis of regression coefficients for the relationship between Fusarium head blight and deoxynivalenol content of wheat. Phytopathology 96:951-961. 
27. Paulitz, T. C. 1996. Diurnal release of ascospores by Gibberella zeae in inoculated wheat plots. Plant Dis. 80:674-678.

28. Pritsch, C., Muehlbauer, G. J., Bushnell, W., R., Somers, D. A., and Vance, C. P. 2000. Fungal development and induction of defense response genes during early infection of wheat spikes by Fusarium graminearum. Mol. Plant-Microbe Interact. 2:159-169.

29. Rocha, O., Ansari, K., and Doohan, F. M. 2005. Effects of trichothecene mycotoxins on eukaryotic cells: A review. Food Addit. Contam. 22:369378.

30. Ryu, D., and Bullerman, L. B. 1999. Effect of cycling temperatures on the production of deoxynivalenol and zearalenone by Fusarium graminearum NRRL 5883. J. Food. Prot. 62:1451-1455.

31. Schmidt-Heydt, M., Magan, N., and Geisen, R. 2008. Stress induction of mycotoxin biosynthesis genes by abiotic factors. FEMS Microbiol. Lett. 284:142-149.
32. Schroeder, H. W., and Christensen, J. J. 1963. Factors affecting resistance of wheat to scab caused by Gibberella zeae. Phytopathology 53:831-838.

33. Shah, D. A., Molineros, J. E., Paul, P. A., Willyerd, K. T., Madden, L. V., and De Wolf, E. D. 2013. Predicting Fusarium head blight epidemics with weather-driven pre- and post-anthesis logistic regression models. Phytopathology 103:906-919.

34. Sneller, C., Guttieri, M., Paul, P., Costa, J., and Jackwood, R. 2012. Variation for resistance to kernel infection and toxin accumulation in winter wheat infected with Fusarium graminearum. Phytopathology 102:306-314.

35. Willyerd, K. T. 2009. Fusarium head blight disease development and mycotoxin accumulation in wheat. Ph.D. thesis, Department of Plant Pathology, Pennsylvania University, University Park.

36. Windels, C. E. 2000. Economic and social impacts of Fusarium head blight: Changing farms and rural communities in the northern Great Plains. Phytopathology 90:17-21. 